IFAS Extension

\title{
Puréed Foods: High Protein ${ }^{1}$
}

\author{
Jamila R. Lepore and Wendy J. Dahl ${ }^{2}$
}

\section{What is protein and why do we need it?}

Protein is an essential nutrient that our bodies need to operate properly and efficiently. Every cell, tissue, and organ in the body needs a consistent source of protein to maintain optimal health and functioning. For these metabolic processes to occur, proteins are broken down and used by the body, only to be replenished by the foods we eat.

\section{Why is protein important for those needing a puréed diet?}

Most individuals get adequate protein by consuming a regular, balanced diet. However, for those with swallowing problems, a condition known as dysphagia, protein becomes even more important. Individuals with dysphagia tend to consume less food due to frustration or difficulty with chewing and swallowing, decreased appetite, or dislike of a modified- texture diet. Older adults with swallowing problems need to emphasize protein in the diet because of age-related changes to body composition and physiological functions (1). Adequate protein helps to preserve lean body mass, minimize skin fragility, and improve immune function and healing.

\section{How much protein do you need?}

Healthy older adults need $1.0 \mathrm{~g} / \mathrm{kg}$ of protein per day (1). For example, an individual of $150 \mathrm{lb}$ (equal to about $68 \mathrm{~kg}$ ) would need about $68 \mathrm{~g}$ of protein per day. Higher amounts of protein may be necessary for older adults with pressure

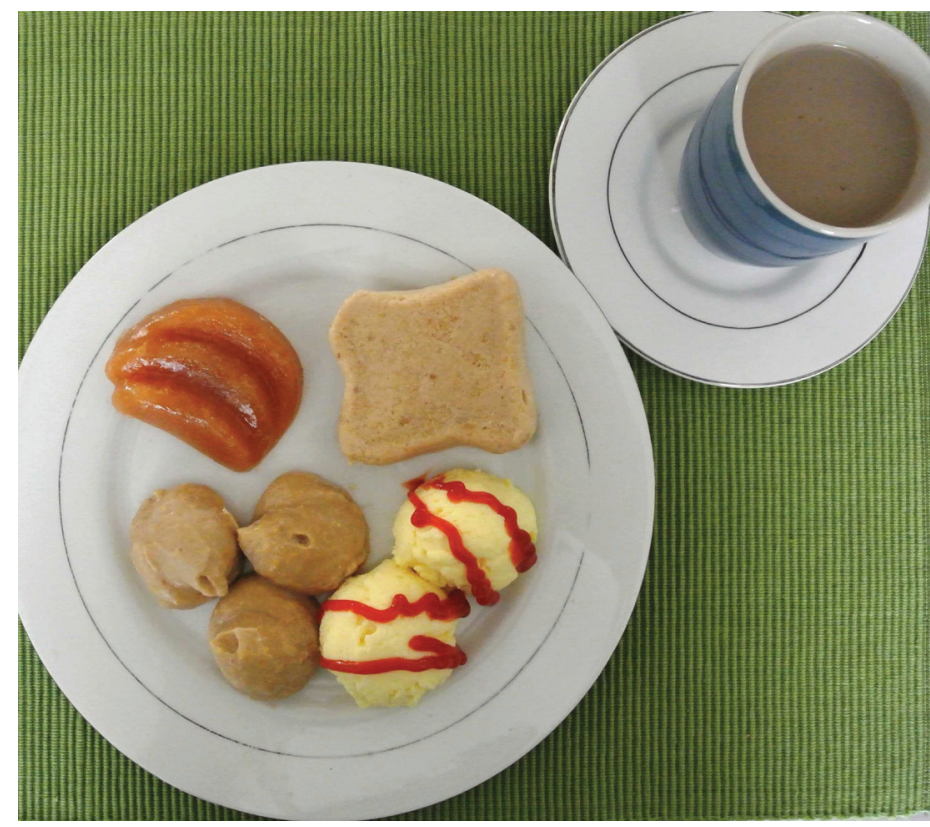

Figure 1. High-protein puréed breakfast Credits: UF/IFAS photo

ulcers, infections, or certain chronic conditions. For more information on individual needs, contact your medical provider or a Registered Dietitian.

For practical purposes, do not be overly concerned with numbers. Instead, aim to incorporate protein into each meal and snack for adequate intake. By providing protein at each meal, a person's daily protein needs can be met. Table 1 indicates the protein contents of common foods.

1. This document is FSHN12-15, one in a series of the Food Science and Human Nutrition Department, Florida Cooperative Extension Service, Institute of Food and Agricultural Sciences, University of Florida. Published March 2013. Visit the EDIS website at http://edis.ifas.ufl.edu/.

2. Jamila R. Lepore, RD, graduate student, and Wendy J. Dahl, PhD, RD, assistant professor; Food Science and Human Nutrition Department, Institute of Food and Agricultural Sciences, University of Florida; Gainesville, FL 32611. 
Table 1. Protein contents of common foods

\begin{tabular}{|l|l|l|}
\hline $\begin{array}{l}\text { Serving } \\
\text { Size }\end{array}$ & Food & $\begin{array}{l}\text { Protein } \\
\text { (grams) }\end{array}$ \\
\hline $3 \mathrm{oz}$ & $\begin{array}{l}\text { Cooked poultry/beef/ } \\
\text { pork/fish }\end{array}$ & $21-25$ \\
\hline $4 \mathrm{oz}$ & $\begin{array}{l}\text { Tofu/tempeh/meatless } \\
\text { burger }\end{array}$ & $10-15$ \\
\hline 2 & $\begin{array}{l}\text { Large eggs } \\
\text { Egg substitute/egg } \\
\text { whites }\end{array}$ & 13 \\
\hline $1 / 2$ cup & $\begin{array}{l}\text { Soft cheese (cottage/ } \\
\text { ricotta) }\end{array}$ & 15 \\
\hline $1 / 2$ cup & Traditional yogurt & $6-8$ \\
\hline $1 / 2$ cup & Greek yogurt & 11 \\
\hline $1 / 2$ cup & Cooked beans (legumes) & $7-9$ \\
\hline $1 / 2$ cup & Cooked soybeans & 11 \\
\hline
\end{tabular}

\section{Animal Protein vs. Plant Protein}

Animal sources of protein, including meat, fish, poultry, eggs, and dairy contain adequate levels of all essential amino acids (building blocks of protein). Plant sources of protein from legumes (beans and lentils) and grains are more nutritious when eaten together. Combining a legume such as beans with a grain, such as rice, makes a protein that is used efficiently by the body. For adults, these combinations do not need to be consumed at the same meal, but should be consumed in the same day (1).

\section{Preparing Puréed Protein Foods Meat and Poultry}

Because of muscle fibers present in meat (beef, pork, lamb, veal, etc.) and poultry, puréeing to a smooth texture can be somewhat tricky. It is important to cook meat to a moist and tender texture. This makes it easier to purée. Meats should be boneless and skinless for ease of preparation. There are two cooking methods that will make puréeing meats easier: braising and stewing. Both techniques include cooking the meat in liquid (for moistness) and cooking for a long time (for tenderness).

\section{BRAISING}

A large cut of meat is partially covered in liquid (such as water, broth, or sauce) and cooked on very low heat for several hours. For more flavor, brown the meat by searing on both sides for a few minutes and then add the liquid for braising.

\section{STEWING}

A large cut of meat is chopped into pieces, covered in liquid, and cooked on very low heat for several hours. It is not necessary to brown the meat with stewing. However, browning adds flavor!

\section{Fish}

Fish generally has a softer texture than meat when cooked, and without added liquid. Fish should be boneless and skinless for ease of preparation. Because most fish is naturally moist and tender, it is best to cook quickly, either by baking, steaming, or frying. Cook fish until it is just cooked through and begins to flake. Over cooking will cause dryness. Cooked fish can be easily puréed in a food processor. Adding tartar sauce or alfredo sauce may make puréed fish more cohesive, smooth, and flavorful.

\section{Canned Meat and Fish}

Canned meat, fish, and poultry are good options for protein purées. This is because these foods have been processed until tender and then packed in water or oil, so they are very moist and often already chopped into small pieces. It is better to drain the liquid before puréeing. This will ensure a higher protein purée with an optimum texture. For example, with tuna or salmon, you should drain the liquid first and then add mayonnaise for a creamy, smooth purée texture.

Examples of Canned Protein Foods: Tuna, Salmon, Crab, Shrimp, Chicken, Sardines

Many condiments and sauces can be used in the preparation of puréed meats and fish. Be as creative as you want, but remember that the flavor must be fantastic! See below for examples of condiments to add to make the purée not only more flavorful but also to create a smoother and more cohesive texture.

\section{Beans, Peas, and Lentils}

Beans and other legumes are good sources of protein and are very easy to purée. This is especially true for canned beans and lentils. Although dry beans may be used as well, be sure to cook until soft before puréeing.

Examples of Common Beans, Peas, and Lentils: Cannellini, Navy, Lima, Pinto, Black, Kidney, Lentils, Soy, Split Peas 
Beans, peas, and lentils provide a source of protein that is often much less expensive than meat, poultry, and fish. Bean, pea, and lentil purées can be used as main course items or as side dishes.

Unlike meat, fish, and poultry, beans, peas, and lentils contain carbohydrate, including fiber. Most people do not get enough fiber. Fiber adds bulk to the stool and enhances bowel regularity. The fiber in beans, peas, and lentils also helps to lower blood cholesterol and blood glucose.

Since beans, peas, and lentils purée so well, preparation time is minimal and texture is usually smooth and cohesive without having to add other ingredients to achieve the recommended "spoon thick" consistency. Beans can be puréed on their own or used as a thickener for other puréed foods, such as soup. See Preparation of Puréed Foods for using beans as a nutritious thickening agent in purées.

Bean, pea, and lentil purées may be more acceptable than meat and poultry purées. First, the texture is smooth and desirable, and usually doesn't have the graininess and grittiness that sometimes occurs with meat and poultry purées. Second, puréed beans are familiar foods that may have been eaten prior to needing a puréed diet. For example, refried beans and hummus are popular puréed foods. Beans can also be combined with puréed meats to achieve good taste, texture and nutrient content.

Soybeans are a great option as they are higher in protein than other beans, and they taste good, too! Soy is also a great option for anyone who wants to avoid or limit meat, and may be a great option for puréed diets. Popular soybased foods include tofu, soy patties, and tempeh. These foods make excellent purées. Soybeans and products made from them tend to be superior texturally compared to many meat purées. This makes them generally more acceptable.

Beans, peas, and lentils are versatile and nutritious, which make them a great addition to a puréed diet.

\section{Dairy and Eggs}

Dairy is one of the best options for puréed proteins as many dairy products are naturally puréed. Yogurt, pudding, and custard are suitable purée consistencies.

Thin dairy products such as milk may be appropriate for some individuals with swallowing problems, but for others, milk may require thickening. While thickened milk is appropriate to use as a source of protein, it is less acceptable than naturally thick-yogurt, pudding, or custard.
Examples of Dairy Products High in Protein: Greek Yogurt, Plain Yogurt, Soft Cheeses (cottage, ricotta)

A pasteurized egg product is another great option for puréed protein foods, and will purée best when scrambled or made into a puréed quiche, frittata, or soufflé. For individuals requiring a low-cholesterol diet, pasteurized egg whites or egg substitutes may be used in place of the egg product.

\section{Making puréed protein foods more flavorful and improving texture}

Sauces, gravies, and condiments may be added to protein dishes to not only make the flavor and overall acceptability better, but also to improve texture. For instance, savory items such as meat may be puréed with a gravy or sauce to create a smoother mouth feel and a better tasting product. Yogurt or cottage cheese may be puréed with fruits or syrups to make a sweet treat that is both nutritious and appealing. See below for common examples of condiments used in both savory and sweet dishes for puréed protein foods.

Ideas for Adding Savory Flavor: Sauce (tomato, BBQ, Alfredo, soy, teriyaki, chili, tartar, curry, etc.); Gravy; Dressing (vinaigrettes, ranch, honey Dijon, etc.); Ketchup, mustard, mayonnaise, sour cream; Vinegar; Juice (lemon, lime); Oil, butter; Broth (chicken, beef, vegetable)

Ideas for Adding Sweetness: Syrup (maple, chocolate, caramel); Jam/jelly; Whipped cream; Fruit purées (applesauce, canned pumpkin); Juice

There are many other ingredients that can enhance the flavor and appeal of puréed foods. This list is simply to give you an idea of the types of products that can be used to improve the acceptability of protein purées.

The sauce you choose will depend on the flavors you want for a particular recipe. It will also depend on the protein item you select. Some of these condiments may provide both flavor and texture improvement. Others may only provide additional flavor, and other items would need to be added to achieve the proper consistency. It is important to have standardized recipes and processing techniques to make sure the puréed proteins not only taste good but are smooth, cohesive, and without lumps. See Preparation of Puréed Foods and Sensory Acceptability of Puréed Foods for how to make purées that are both safe and appetizing. 
For meat and poultry products specifically, there are several items that can be added to reduce the gritty and grainy texture that sometimes exists with these purées. It can also be difficult to get meat and poultry to form a cohesive and smooth texture. The addition of the foods listed below can improve both of these attributes.

Improving Cohesiveness and Texture of Meat and Poultry Purées: Puréed beans; Condiments; Sour cream, mayonnaise, yogurt, ricotta/cottage cheese; Sauce/Gravy; Creamy dressings

Even when meats are moist and tender before puréeing, the texture once puréed is not always as smooth and creamy as desired.

While any of these products will work with the purée, the best way to improve the texture of meat and poultry is to add puréed beans. The addition of puréed beans not only makes the texture more consistent, it adds a great source of protein, fiber, vitamins, and minerals. Also, beans will not alter the flavor of the original purée as much as condiments would. By using beans, chicken will more likely taste like chicken than if gravy were added to it!

Condiments such as sauces and gravies have their place in purées as well. However, these do not usually provide the nutritional value that beans do and will change the flavor, which may or may not be desirable. Remember, too, that condiments may be added as a garnish over the purée instead of being mixed in. For example, create a chicken purée made with puréed navy beans and pour thickened gravy on top of the purée to make it more appealing. To learn more about garnishes and how to improve the appearance and flavor of purées, see Sensory Acceptability of Puréed Foods.

\section{Summary and Useful Links}

Protein is an important nutrient. Many older adults do not consume enough of this vital nutrient, crucial to overall health and well-being. For those with dysphagia and on a puréed diet, consuming sufficient protein is even more difficult. Be sure to include high-protein purées at each meal and with snacks. As puréed protein foods such as beef and poultry may be less desirable, it is important to optimize the acceptance of these foods.

For optimum flavor, textures, and nutrition of meat and poultry, use a combination of puréed beans and condiments or garnishes when preparing these purées. Choose puréed beans, dairy, and eggs more often. These foods are generally acceptable since many are traditionally prepared as purées or occur naturally as purées.

Finally, we could make the most nutritious puréed protein food possible, but if it doesn't look, smell and taste delicious, it won't be eaten! While a texture-modified diet may not be the diet of choice, the goal is to make it as appealing as possible so that the person consuming the purées can experience a better quality of life.

\section{For more information, related EDIS publications are available at http://edis.ifas.ufl.edu/:}

- Puréed Foods for Swallowing Problems (2)

- Swallowing Problems and the Older Adult (3)

- Sensory Acceptability of Puréed Foods

- Preparation of Puréed Foods

\section{References}

(1) Kaiser M, Bandinelli S, Lunenfeld B. Frailty and the role of nutrition in older people. A review of the current literature. Acta Biomed. 2010;81 Suppl 1:37-45.

(2) Dahl WJ. Puréed foods for swallowing problems. 2011 http://edis.ifas.ufl.edu/pdffiles/FS/FS16800.pdf

(3) Dahl WJ. Swallowing Problems and the Older Adult. 2011 http://edis.ifas.ufl.edu/pdffiles/FS/FS16400.pdf 